\title{
Expression analysis of miRNA and target mRNAs in esophageal cancer
}

\author{
X.R. Meng ${ }^{1 *}$, P. Lü ${ }^{2 *}$, J.Z. $\mathrm{Mei}^{3}$, G.J. Liu $^{3}$ and Q.X. Fan ${ }^{1}$ \\ ${ }^{1}$ Oncology Department, The First Affiliated Hospital of Zhengzhou University, Zhengzhou, China \\ ${ }^{2}$ Gastrointestinal Surgery Department, People's Hospital of Zhengzhou, Zhengzhou, China \\ ${ }^{3}$ Medical Oncology Department, People's Hospital of Zhengzhou, Zhengzhou, China
}

\begin{abstract}
We aimed to investigate miRNAs and related mRNAs through a network-based approach in order to learn the crucial role that they play in the biological processes of esophageal cancer. Esophageal squamous-cell carcinoma (ESCC) and adenocarcinoma (EAC)-related miRNA and gene expression data were downloaded from the Gene Expression Omnibus database, and differentially expressed miRNAs and genes were selected. Target genes of differentially expressed miRNAs were predicted and their regulatory networks were constructed. Differentially expressed miRNA analysis selected four miRNAs associated with EAC and ESCC, among which $h s a-m i R-21$ and $h s a-m i R-202$ were shared by both diseases. hsa-miR-202 was reported for the first time to be associated with esophageal cancer in the present study. Differentially expressed miRNA target genes were mainly involved in cancer-related and signal-transduction pathways. Functional categories of these target genes were related to transcriptional regulation. The results may indicate potential target miRNAs and genes for future investigations of esophageal cancer.
\end{abstract}

Key words: Esophageal adenocarcinoma; Esophageal squamous-cell carcinoma; miRNA expression network; Pathway; Gene ontology

\section{Introduction}

Esophageal cancer, with squamous-cell carcinoma (ESCC) and adenocarcinoma (EAC) as the predominant histological types, is the sixth leading cause of cancerrelated mortality and the eighth most common cancer worldwide. The overall 5-year survival ranges from 15\% to $25 \%$, and the best outcomes are associated with disease diagnosed in the early stages (1). MicroRNAs (miRNAs) are an abundant class of small nonprotein-coding RNAs that function as negative gene regulators. miRNAs have gained significant attention because of their ability to regulate multiple oncogene and tumor suppressor signaling pathways (2). Evidence that alterations in the expression of certain miRNAs (e.g., hsa-miR-21, hsa-miR-223 and hsamiR-75) might be associated with the development, prognosis and survival rates of esophageal cancer is increasing (3). However, most previous studies have focused mainly on differences in expression of single miRNAs instead of focusing on the miRNAs and the specifically regulated $m R N A s$ through a view of the network that plays a crucial role in the whole biological process (4).
This study was designed to investigate the pathogenesis of ESCC and EAC by 1) screening existing EAC- and ESCC-related miRNA expression microarray data to identify differentially expressed miRNAs and analyze the correlations between miRNA expression and the risk factors, treatment methods and survival rates of patients; 2) screening the EAC- and ESCC-related gene expression microarray data for differentially expressed genes; 3) predicting the target genes of differentially expressed miRNAs and constructing regulatory networks depending on the differentially expressed target genes. Their effects on biological processes of the target genes were also investigated with pathway and gene ontology (GO) enrichment analysis.

\section{Material and Methods}

\section{Databases}

We downloaded EAC- and ESCC-related miRNA microarray data and gene expression microarray data

Correspondence: Q.X. Fan, Oncology Department, The First Affiliated Hospital, Zhengzhou University, Zhengzhou, China. E-mail: fqx2243@126.com

${ }^{*}$ These authors contributed equally to this study.

Received February 7, 2014. Accepted May 19, 2014. First published online August 1, 2014. 
Table 1. Differentially expressed miRNAs in esophageal adenocarcinoma (EAC) and squamous-cell carcinoma (ESCC).

\begin{tabular}{lrcc}
\hline miRNAs & FC & $\mathrm{P}$ & FDR \\
\hline EAC & & & \\
hsa-miR-21 & 1.12 & $1.47 \mathrm{E}-04$ & 0.023 \\
hsa-miR-202 & -1.81 & $1.02 \mathrm{E}-04$ & 0.023 \\
hsa-miR-203 & -2.71 & $1.35 \mathrm{E}-04$ & 0.023 \\
hsa-miR-205 & -2.45 & $3.12 \mathrm{E}-04$ & 0.041 \\
ESCC & & & \\
hsa-miR-21 & 1.41 & $2.07 \mathrm{E}-10$ & $2.37 \mathrm{E}-07$ \\
hsa-miR-202 & -1.64 & $1.81 \mathrm{E}-04$ & $5.57 \mathrm{E}-03$ \\
hsa-miR-223 & 1.25 & $3.74 \mathrm{E}-06$ & $4.06 \mathrm{E}-04$ \\
hsa-miR-375 & -1.40 & $1.01 \mathrm{E}-06$ & $1.42 \mathrm{E}-04$ \\
\hline
\end{tabular}

FC: fold change; FDR: false discovery rate. The $t$-test was used for statistical analyses.

from the Gene Expression Omnibus (GEO) database. Gene Ontology (GO) analysis and Kyoto Encyclopedia of Gene and Genome (KEGG) pathway enrichment analysis were used to define functions and pathways in ESCC and EAC. A protein-protein interaction network was constructed from the Biomolecular Interaction Network Database (BIND) to identify modules with close interactions.

\section{Study samples}

miRNA analysis. We performed a comprehensive miRNA analysis to identify differential miRNA using the miRNA profile (GSE13937), which was carried out using the OSU-CCC Human MicroRNA Microarray Version 3.0 Array (http://www.ncbi.nlm.nih.gov/geo/query/acc.cgi?acc = GPL8835), and included data from 76 esophageal cancer cases (44 ESCC and 32 EAC) and 76 adjacent noncancerous tissues.

Gene expression analysis. The gene expression microarray data was based on the profile via the Affymetrix
Human Genome U133 Plus 2.0 Array (HG-U133_Plus_2). The profile included four collections: GSE42363 (14 EAC tissue samples), GSE17351 (5 ESCC cases and 5 adjacent normal esophageal tissues), GSE26886 (21 EAC tissues, 9 ESCC tissues, and 19 normal tissues from healthy subjects), and GSE3701 (40 ESCC tissue samples).

\section{Statistical analyses}

Differential expression analysis. Raw data including Affymetrix CEL files and simple omnibus format in text files for all samples as described above were obtained from the GEO database. Raw intensity values generated from the CEL files were normalized by robust multiarray analysis (RMA) (5) as follows. Firstly, background noise and the processing artifacts were neutralized using a model-based background correction. Secondly, expression values were normalized by aligning to a common scale. Thirdly, an expression value was generated for each probe using an iterative median polishing method. The resulting $\log _{2}-$ transformed RMA expression values were then used to further identify significantly differentially expressed genes and miRNAs. The $t$-test was used to identify differentially expressed genes, and the Benjamini and Hochberg procedure (6) was carried out for multiple test corrections. The genes or miRNAs with a false discovery rate (FDR) $<0.05$ were selected as differentially expressed. Differentially expressed genes or miRNAs were identified as up- or down-regulated according to the fold-change value. All the above procedures were performed using the R software (v3.03, http://www.r-project.org/) with BioConductor (http:// www.bioconductor.org/), linear models for microarray (limma) data packages (3.12.1) and libraries (7), and differentially co-expressed genes and links packages (8).

miRNA target prediction. miRNA target sites in 3' UTR gene regions were identified by bioinformatics analysis using the Miranda (microRNA.org), microcode (http://www. mircode.org/mircode/), MirTarget2 (9), Targetscan (http://
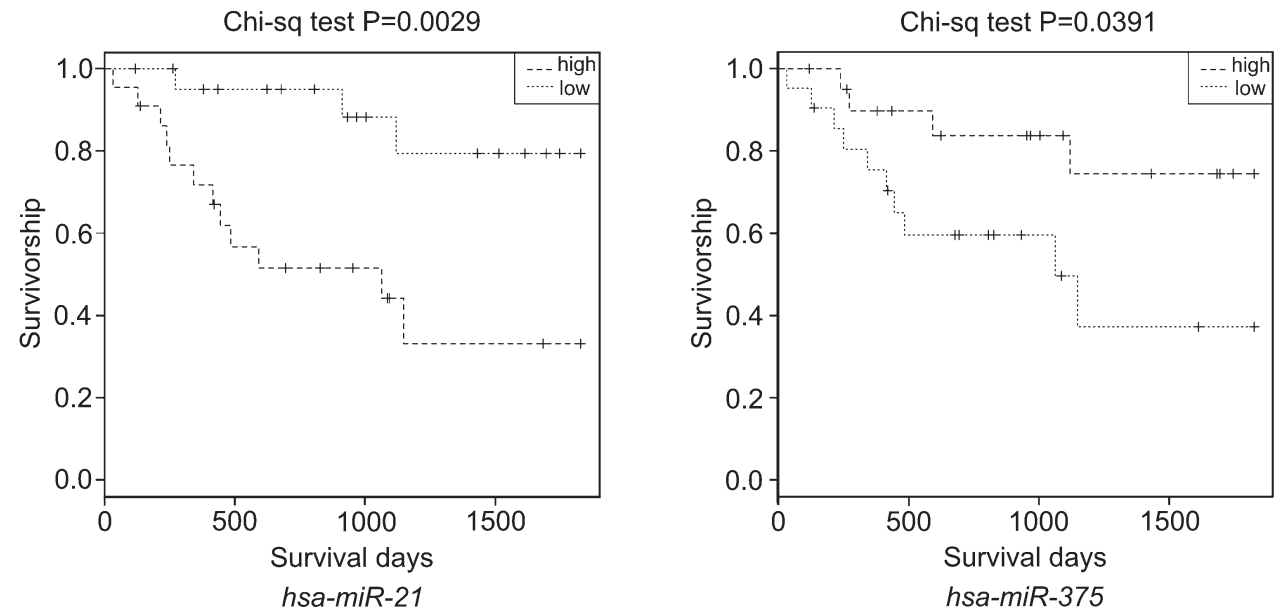

Figure 1. Survival analyses of $h s a-m i R-21$ and $h s a-m i R-375$ in esophageal squamous-cell carcinoma patients. 


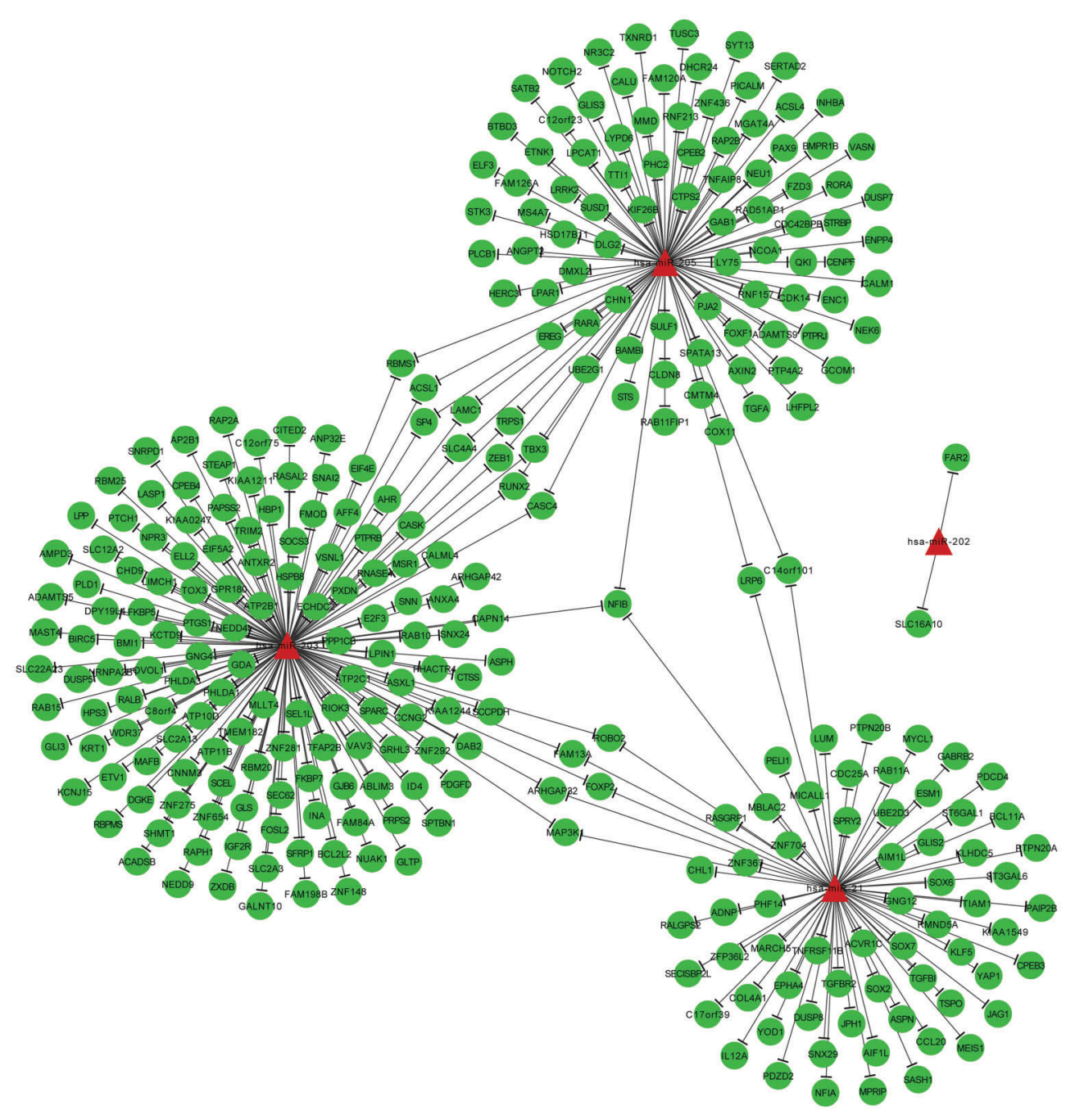

Figure 2. Regulatory network of selected miRNAs and their differentially expressed target genes in esophageal adenocarcinoma.

www.targetscan.org/), PicTar (http://pictar.mdc-berlin.de/) and microT (http://diana.cslab.ece.ntua.gr/microT/) databases. Only those putative miRNA target sites resulting from at least three databases were considered positive.

Enrichment analysis. To capture biologically relevant signatures of the differentially expressed target genes, we carried out enrichment analyses. All dysregulated target genes were mapped to the KEGG pathways (http://www. genome.jp/kegg/) database (10) and the GO database (11). The hypergeometric distribution test was used to identify biological processes significantly enriched with differentially expressed target genes.

\section{Results}

\section{Differentially expressed miRNAs in esophageal cancer tissues}

Compared with the normal tissues, EAC tissues exhibited 4 differentially expressed miRNAs. One, hsa-miR-21, was upregulated and the other three (hsa-miR-202, hsa-miR-203, and hsa-miR-205) were downregulated. In ESCC, the expression levels of hsa-miR-21 and hsa-miR223 were elevated compared with the normal tissue, while those of hsa-miR-202 and hsa-miR-375 were decreased (Table 1).

In EAC, the expression levels of the 4 differentially expressed miRNAs had no significant correlation with drinking, smoking, treatment methods or survival time of the patients $(P>0.05)$. However, in ESCC, the expression level of hsa-miR-21 showed a significant negative correlation with the survival time of the patients $(P=0.0029$, Figure 1). In ESCC patients, hsa-miR-375 was positively associated with survival time $(P=0.0391$, Figure 1$)$.

\section{Differentially expressed genes in esophageal cancer tissues}

Compared with the normal tissues, 641 downregulated genes and 628 upregulated genes were detected in 


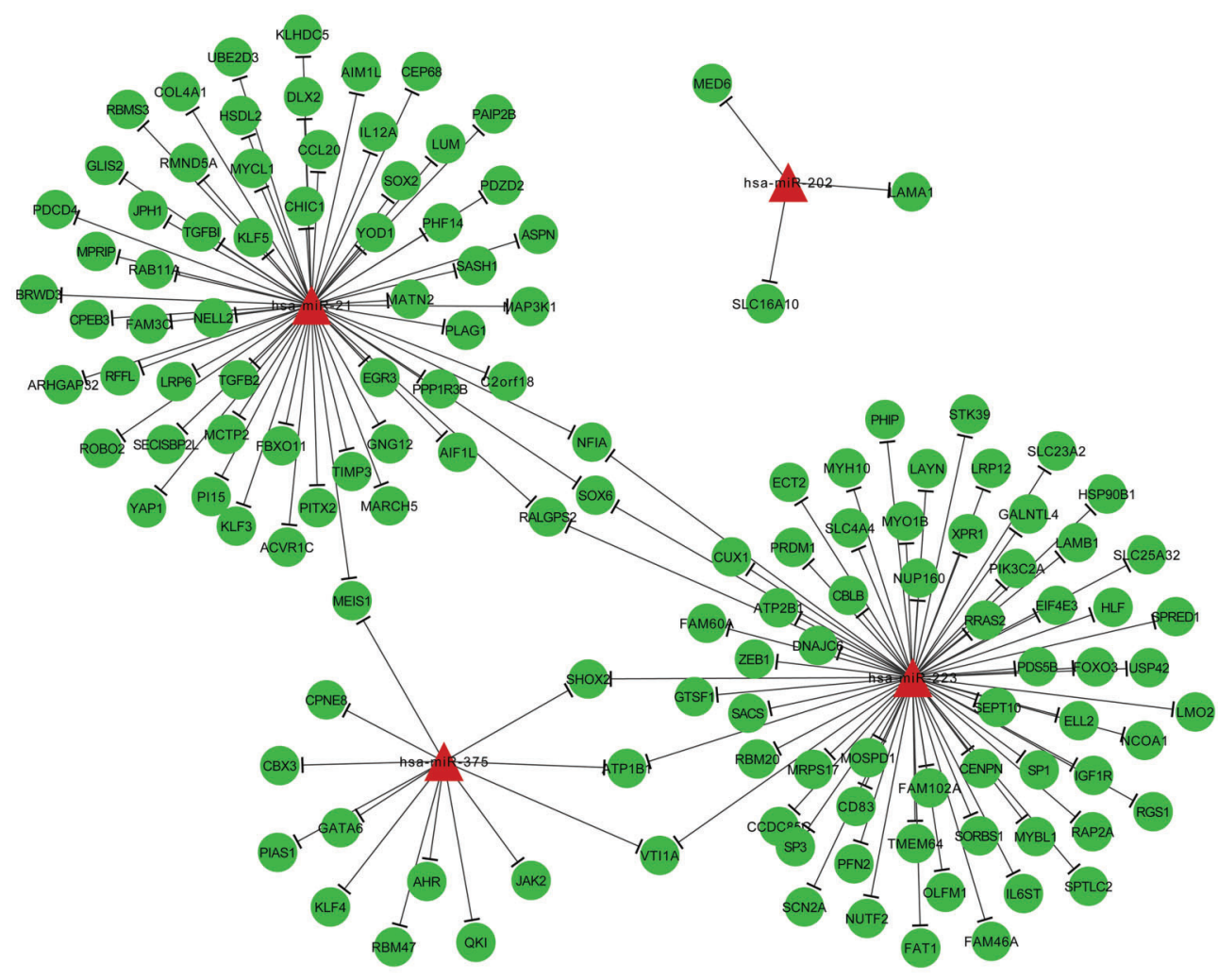

Figure 3. Regulatory network of selected miRNAs and their differentially expressed target genes in esophageal squamous-cell carcinoma.

the EAC samples. There were 1110 differentially expressed genes (516 with decreased expression and 594 with increased expression) detected in ESCC samples compared with their normal counterparts.

\section{Differentially expressed miRNAs and their target genes prediction}

As noted, there were 4 differentially expressed miRNAs (hsa-miR-21, hsa-miR-202, hsa-miR-203 and hsa-miR-205) in EAC samples, and 4 (hsa-miR-21, hsamiR-202, hsa-miR-223 and hsa-miR-375) in ESCC samples. We tried to predict the target genes of each differentially expressed miRNA and construct their networks (Figures 2 and 3). For EAC, we screened 71 target genes for hsa-miR-21, 2 for hsa-miR-202, 152 for hsamiR-203 and 98 for hsa-miR-205. In ESCC, 59 genes were screened as the target genes for hsa-miR-21, 3 for hsa-miR-202, 65 for hsa-miR-223 and 13 for hsa-miR375. Furthermore, we found some genes that were the target genes of more than one differentially expressed miRNA. For example, NFIB was the target gene of hsamiR-21, hsa-miR-203 and hsa-miR-205 in EAC, and ATP1B for hsa-miR-223 and hsa-miR-375 in ESCC.

We carried out KEGG pathway and GO enrichment analysis of the differentially expressed target genes in both
EAC and ESCC. In EAC samples, 11 pathways were enriched in differentially expressed target genes, with the Hippo signaling pathway (hsa04290) as the most significant one (Table 2). Five of the remaining pathways, microRNAs in cancer (hsa05206), pancreatic cancer (hsa05212), pathways in cancer (hsa05200), basal cell carcinoma (hsa05217) and colorectal cancer (hsa05210), were reported as correlated with cancer. GO enrichment analysis revealed $3 \mathrm{GO}$ items overrepresented with dysregulated target genes of the selected miRNAs, and these were items mainly involved in the transcription process (Table 3 ).

For ESCC, there were also 11 pathways (Table 2) that demonstrated enrichment of differentially expressed target genes, with the Jak-STAT signaling pathway (hsa04630) related to signal transduction as the most remarkable one. Two cancer-related pathways, proteoglycans in cancer (hsa05205) and transcriptional misregulation in cancers (hsa05202), were also indicated. GO enrichment analysis revealed 7 items enriched with dysregulated target genes (Table 3). Five of the seven items were also related to the transcription process.

\section{Discussion}

The results of differentially expressed miRNA indicated 
Table 2. KEGG pathway enrichment analysis for the differentially expressed target genes of selected miRNAs in esophageal adenocarcinoma (EAC) and squamous-cell carcinoma (ESCC).

\begin{tabular}{|c|c|c|c|}
\hline KEGG_id & Pathway description & Pathway subclass & $P$ \\
\hline \multicolumn{4}{|l|}{ EAC } \\
\hline hsa04390 & Hippo signaling pathway & Signal transduction & 4.84E-04 \\
\hline hsa05206 & MicroRNAs in cancer & Cancers & 3.63E-03 \\
\hline hsa04350 & TGF-beta signaling pathway & Signal transduction & 7.15E-03 \\
\hline hsa05212 & Pancreatic cancer & Cancers & $1.48 \mathrm{E}-02$ \\
\hline hsa04961 & Endocrine and other factor-regulated calcium reabsorption & Excretory system & 1.66E-02 \\
\hline hsa05200 & Pathways in cancer & Cancers & $2.49 \mathrm{E}-02$ \\
\hline hsa04120 & Ubiquitin mediated proteolysis & Folding, sorting and degradation & 3.43E-02 \\
\hline hsa00564 & Glycerophospholipid metabolism & Lipid metabolism & 3.81E-02 \\
\hline hsa05217 & Basal cell carcinoma & Cancers & 3.99E-02 \\
\hline hsa04972 & Pancreatic secretion & Digestive system & 4.62E-02 \\
\hline hsa05210 & Colorectal cancer & Cancers & 4.91E-02 \\
\hline \multicolumn{4}{|l|}{ ESCC } \\
\hline hsa04630 & Jak-STAT signaling pathway & Signal transduction & 4.28E-04 \\
\hline hsa04961 & Endocrine and other factor-regulated calcium reabsorption & Excretory system & $5.28 \mathrm{E}-03$ \\
\hline hsa05145 & Toxoplasmosis & Infectious diseases & 8.36E-03 \\
\hline hsa05205 & Proteoglycans in cancer & Cancers & $1.11 \mathrm{E}-02$ \\
\hline hsa04964 & Proximal tubule bicarbonate reclamation & Excretory system & 1.34E-02 \\
\hline hsa04972 & Pancreatic secretion & Digestive system & $1.40 \mathrm{E}-02$ \\
\hline hsa04350 & TGF-beta signaling pathway & Signal transduction & 2.30E-02 \\
\hline hsa04120 & Ubiquitin mediated proteolysis & Folding, sorting and degradation & 2.66E-02 \\
\hline hsa04976 & Bile secretion & Digestive system & 3.14E-02 \\
\hline hsa04151 & PI3K-Akt signaling pathway & Signal transduction & $3.18 \mathrm{E}-02$ \\
\hline hsa05202 & Transcriptional misregulation in cancers & Cancers & 4.07E-02 \\
\hline
\end{tabular}

The hypergeometric distribution test was used for statistical analyses.

Table 3. Gene ontology (GO) enrichment analysis for the differentially expressed target genes of selected miRNAs in esophageal adenocarcinoma (EAC) and squamous-cell carcinoma (ESCC).

\begin{tabular}{lccc}
\hline GO_id & GO_description & GO_class & P \\
\hline EAC & Positive regulation of transcription, DNA-dependent & Process & $1.07 \mathrm{E}-02$ \\
GO:0045893 & Negative regulation of transcription, DNA-dependent & Process & $1.94 \mathrm{E}-02$ \\
GO:0045892 & Positive regulation of transcription from RNA polymerase II & Process & $3.02 \mathrm{E}-02$ \\
GO:0045944 & promoter & & \\
ESCC & Positive regulation of transcription from RNA & Process & $2.35 \mathrm{E}-03$ \\
GO:0045944 & polymerase II promoter & $6.03 \mathrm{E}-03$ \\
GO:0003700 & Sequence-specific DNA binding transcription factor activity & Function & $2.23 \mathrm{E}-02$ \\
GO:0005667 & Transcription factor complex & Component & $3.49 \mathrm{E}-02$ \\
GO:0045893 & Positive regulation of transcription, DNA-dependent & Process & $4.62 \mathrm{E}-02$ \\
GO:0044212 & Transcription regulatory region DNA binding & Function & $4.99 \mathrm{E}-02$ \\
GO:0003682 & Chromatin binding & Function & $4.99 \mathrm{E}-02$ \\
GO:0005606 & Laminin-1 complex & Component & \\
\hline
\end{tabular}

The hypergeometric distribution test was used for statistical analyses. 
elevated expression of hsa-miR-21 and decreased expression of hsa-miR-202 in both EAC and ESCC sample tissues. Saad and colleagues reported increased hsa-miR-21 expression in EAC in a sample of $34 \mathrm{EAC}$ and 46 normal tissues, which was subsequently confirmed by RT-PCR (12). At the same time, they also found reduced hsa-miR-203 and hsa-miR-205 expression in EAC tissues, which is consistent with the present study, and has also been confirmed by RT-PCR. Moreover, the clinical pathological characteristics analysis of this study showed that the low expression of hsa-miR-203 was significantly associated with process and stage of EAC (12). Hsa-miR-205 is thought to play a role in inhibiting cancer in the process of EAC formation (13); therefore the decreased expression may promote tumor occurrence. Up to now, no previous study has reported the differential expression of $h s a-m i R-202$ in correlation with esophageal cancer. However, hsa-miR-202 has been confirmed related to other cancers, such as breast cancer (14) and gastric cancer (15). Hsa-miR-21 was also found to be excessively expressed in ESCC tissues in other studies $(16,17)$. It was reported that the increased expression of hsa-miR-223 in ESCC might influence the expression of gene FBXW7 (18), which affected the prognosis of patients. Therefore hsamiR-223 has also been regarded as a reliable diagnostic biomarker of ESCC (19). MiR-375 has been seen as a tumor suppressor molecule, modulating $L D H B$ action to curb the occurrence of tumor development (20), which is consistent with the hsa-miR-375 result in this study. The miRNA result revealed a significant negative correlation of hsa-miR-21 expression and positive correlation with ESCC survival time, which is consistent with a previous study (21) and indicates that hsa-miR-21 and hsa-miR-375 might be reliable prognostic markers of ESCC.

We predicted the target genes of the differentially expressed EAC and ESCC miRNAs, constructed their networks, and carried out enrichment pathway analysis of target genes. Among the differentially expressed miRNAs,

\section{References}

1. Pennathur A, Gibson MK, Jobe BA, Luketich JD. Oesophageal carcinoma. Lancet 2013; 381: 400-412, doi: 10.1016/S0140-6736(12)60643-6.

2. Esquela-Kerscher A, Slack FJ. Oncomirs - microRNAs with a role in cancer. Nat Rev Cancer 2006; 6: 259-269, doi: $10.1038 / \mathrm{nrc} 1840$.

3. Ogawa R, Ishiguro $H$, Kuwabara $Y$, Kimura M, Mitsui A, Katada $\mathrm{T}$, et al. Expression profiling of micro-RNAs in human esophageal squamous cell carcinoma using RTPCR. Med Mol Morphol 2009; 42: 102-109, doi: 10.1007/ s00795-009-0443-1.

4. Stelzl U, Worm U, Lalowski M, Haenig C, Brembeck FH, Goehler $\mathrm{H}$, et al. A human protein-protein interaction network: a resource for annotating the proteome. Cell 2005; 122: 957-968, doi: 10.1016/j.cell.2005.08.029.

5. Irizarry RA, Hobbs B, Collin F, Beazer-Barclay YD,
hsa-miR-202 was for the first time reported to be correlated with both EAC and ESCC. The Network diagram indicated that the differentially expressed target genes of hsa-miR-202 were FAR2 and SLC16A10 for EAC, and MED6, SLC16A10 and LAMA1 for ESCC. None of these genes had been previously mentioned in relation with EAC or ESCC.

Five of the 11 pathways enriched for EAC are known to be cancer-related, and two, the Hippo signaling pathway (hsa04390) and the transforming growth factor (TGF)-betasignaling pathway (hsa04350), are signal-transduction pathways. The Hippo signaling pathway is associated with other key signaling pathways such as the TGF-beta and Wnt mediated pathways, and has been reported to be associated with cancer (22). Previous studies have confirmed that the TGF-beta-signaling pathway promotes the development of EAC by activating Notch signaling and SOX9 gene function (23). The most significantly target gene-enriched ESCC pathways were also signal transduction pathways: the Jak-STAT signaling pathway (hsa04630), TGF-beta signaling pathway (hsa04350), and PI3K-Akt signaling pathway (hsa04151). All three are known to be correlated with the development of ESCC (24). GO enrichment analysis revealed abnormal regulation of the transcription process in both EAC and ESCC, which may explain the clinical similarity of the two diseases.

Differentially expressed miRNA analysis selected 4 miRNAs associated with EAC and ESCC, among which hsa-miR-21 and hsa-miR-202 were shared by both diseases. hsa-miR-202 was reported for the first time to be correlated with esophageal cancer in the present study. The pathway analysis of miRNA target genes suggested that differentially expressed miRNA target genes were mainly involved in cancer-related and signal-transduction pathways. Functional categories of these target genes were related to transcriptional regulation. Our results indicated potential target miRNAs for future therapeutic investigations.
Antonellis KJ, Scherf U, et al. Exploration, normalization, and summaries of high density oligonucleotide array probe level data. Biostatistics 2003; 4: 249-264, doi: 10.1093/ biostatistics/4.2.249.

6. Benjamini $Y$, Hochberg Y. Controlling the false discovery rate: a practical and powerful approach to multiple testing. $J$ Royal Stat Soc Series B (Methodological) 1995; 57: 289-300.

7. Smyth GK, Michaud J, Scott HS. Use of within-array replicate spots for assessing differential expression in microarray experiments. Bioinformatics 2005; 21: 20672075, doi: 10.1093/bioinformatics/bti270.

8. Liu BH, Yu H, Tu K, Li C, Li YX, Li YY. DCGL: an R package for identifying differentially coexpressed genes and links from gene expression microarray data. Bioinformatics 2010; 26: 2637-2638, doi: 10.1093/bioinformatics/btq471.

9. Wang X, El Naqa I. Prediction of both conserved and 
nonconserved microRNA targets in animals. Bioinformatics 2008; 24: 325-332, doi: 10.1093/bioinformatics/btm595.

10. Kanehisa M, Goto S. KEGG: kyoto encyclopedia of genes and genomes. Nucleic Acids Res 2000; 28: 27-30, doi: 10.1093/nar/28.1.27.

11. Ashburner $M$, Ball CA, Blake JA, Botstein $D$, Butler $H$, Cherry JM, et al. Gene ontology: tool for the unification of biology. The Gene Ontology Consortium. Nat Genet 2000; 25: 25-29, doi: 10.1038/75556.

12. Saad R, Chen Z, Zhu S, Jia P, Zhao Z, Washington MK, et al. Deciphering the unique microRNA signature in human esophageal adenocarcinoma. PLoS One 2013; 8: e64463, doi: $10.1371 /$ journal.pone.0064463.

13. Wu X, Ajani JA, Gu J, Chang DW, Tan W, Hildebrandt MA, et al. MicroRNA expression signatures during malignant progression from Barrett's esophagus to esophageal adenocarcinoma. Cancer Prev Res 2013; 6: 196-205, doi: 10.1158/1940-6207.CAPR-12-0276.

14. Iorio MV, Ferracin M, Liu CG, Veronese A, Spizzo R, Sabbioni S, et al. MicroRNA gene expression deregulation in human breast cancer. Cancer Res 2005; 65: 7065-7070, doi: 10.1158/0008-5472.CAN-05-1783.

15. Jiang Z, Guo J, Xiao B, Miao Y, Huang R, Li D, et al Increased expression of miR-421 in human gastric carcinoma and its clinical association. J Gastroenterol 2010; 45: 17-23, doi: 10.1007/s00535-009-0135-6.

16. Mori $Y$, Ishiguro $H$, Kuwabara $Y$, Kimura M, Mitsui A, Ogawa $\mathrm{R}$, et al. MicroRNA-21 induces cell proliferation and invasion in esophageal squamous cell carcinoma. Mol Med Rep 2009; 2: 235-239.

17. Nouraee N, Van Roosbroeck K, Vasei M, Semnani S, Samaei NM, Naghshvar F, et al. Expression, tissue distribution and function of miR-21 in esophageal squamous cell carcinoma. PLoS One 2013; 8: e73009, doi: 10.1371/ journal.pone.0073009.

18. Kurashige J, Watanabe M, Iwatsuki M, Kinoshita K, Saito S, Hiyoshi $Y$, et al. Overexpression of microRNA-223 regulates the ubiquitin ligase FBXW7 in oesophageal squamous cell carcinoma. Br J Cancer 2012; 106: 182-188, doi: 10.1038/ bjc.2011.509.

19. Zhang C, Wang C, Chen X, Yang C, Li K, Wang J, et al. Expression profile of microRNAs in serum: a fingerprint for esophageal squamous cell carcinoma. Clin Chem 2010; 56: 1871-1879, doi: 10.1373/clinchem.2010.147553

20. Isozaki $\mathrm{Y}$, Hoshino I, Nohata N, Kinoshita T, Akutsu $\mathrm{Y}$, Hanari $\mathrm{N}$, et al. Identification of novel molecular targets regulated by tumor suppressive miR-375 induced by histone acetylation in esophageal squamous cell carcinoma. Int $J$ Oncol 2012; 41: 985-994.

21. Komatsu S, Ichikawa D, Takeshita H, Konishi H, Nagata $H$, Hirajima $S$, et al. Prognostic impact of circulating miR-21 and miR-375 in plasma of patients with esophageal squamous cell carcinoma. Expert Opin Biol Ther 2012; 12 (Suppl 1): S53-S59, doi: 10.1517/14712598.2012.681373.

22. Liu AM, Wong KF, Jiang X, Qiao Y, Luk JM. Regulators of mammalian Hippo pathway in cancer. Biochim Biophys Acta 2012; 1826: 357-364.

23. Song S, Maru DM, Ajani JA, Chan CH, Honjo S, Lin HK, et al. Loss of TGF-beta adaptor beta2SP activates notch signaling and SOX9 expression in esophageal adenocarcinoma. Cancer Res 2013; 73: 2159-2169, doi: 10.1158/ 0008-5472.CAN-12-1962.

24. You Z, Xu D, Ji J, Guo W, Zhu W, He J. JAK/STAT signal pathway activation promotes progression and survival of human oesophageal squamous cell carcinoma. Clin Trans/ Oncol 2012; 14: 143-149, doi: 10.1007/s12094-012-0774-6. 